\title{
EFEITO ANTICARCINOGÊNICO DO GERIOOX® EM Drosophila melanogaster
}

\author{
Nayara Júnia de Souza Bontempo '; Priscila Capelari Orsolin ${ }^{2}$
}

1 Graduanda em Medicina Veterinária pelo Centro Universitário de Patos de Minas (UNIPAM), Patos de Minas, Minas Gerais, Brasil

(email para correspondência: nayarajunia@hotmail.com)

2 Docente do Centro Universitário de Patos de Minas (UNIPAM), mestre e doutora em Genética e Bioquímica pela Universidade Federal de Uberlândia (UFU), com ênfase em Mutagênese e Carcinogênese, Patos de Minas, Minas Gerais, Brasil

Recebido em: 02/10/2017 - Aprovado em: 21/11/2017 - Publicado em: 05/12/2017 DOI: 10.18677/EnciBio_2017B27

\begin{abstract}
O Gerioox® é um medicamento composto por ômega 3, D-glucosamina, sulfato de condroitina, vitamina $\mathrm{E}$, cobre, zinco e selenito de sódio. É indicado como medicação geriátrica, devido aos seus efeitos antioxidantes, condroprotetores e dermatológicos para cães e gatos, visando melhorar a vitalidade e o estado geral de animais em idade avançada. Alguns trabalhos têm evidenciado que sua utilização pode contribuir na terapêutica do câncer. Desse modo, este estudo foi executado com o objetivo principal de avaliar o possível efeito anticarcinogênico do Gerioox® em Drosophila melanogaster, por meio do teste para detecção de clones de tumores epiteliais (warts). Para isso, prepararam-se três concentrações de Gerioox®: 1, 2 e 4 $\mathrm{mg} / \mathrm{mL}$, que foram utilizadas em associação com doxorrubicina (em sistema de cotratamento) ou isoladamente. O procedimento experimental foi realizado com todas as larvas descendentes do cruzamento de fêmeas wts/TM3 com machos $m w h / m w h$. Os resultados revelaram atividade anticarcinogênica do Gerioox $\circledast$, uma vez que houve redução estatisticamente significativa $(p<0,05)$ nas frequências de tumores observadas nas duas maiores concentrações testadas de Gerioox® em associação à DXR, quando comparadas à frequência de tumores no controle positivo. Conclui-se, portanto, que nas presentes condições experimentais, o Gerioox® reduziu a frequência de tumores induzidos pela doxorrubicina em $D$. melanogaster.
\end{abstract}

RESUMO

PALAVRAS-CHAVE: Anticarcinogênico, Drosophila melanogaster, Gerioox®.

\section{ANTICARCINOGENIC EFFECT OF GERIOOX® IN Drosophila melanogaster}

\begin{abstract}
Gerioox $\circledast$ is a medicine composed of omega 3, D-glucosamine, chondroitin sulfate, vitamin E, copper, zinc and sodium selenium. It is indicated as geriatric medication, due to its antioxidant, chondroprotective and dermatological effects for dogs and cats, aiming at improving the vitality and the general state of the animals in old age. Some studies have shown that its application may contribute to cancer therapy. Thus, this study was performed with the main objective of evaluating the possible anticarcinogenic effect of Gerioox ${ }^{\circledR}$ in Drosophila melanogaster, by means of the test ENCICLOPÉDIA BIOSFERA, Centro Científico Conhecer - Goiânia, v.14 n.26; p.317 2017
\end{abstract}


for the detection of clones of epithelial tumors (warts). For this, three concentrations of Gerioox $\AA$ were prepared: 1,2 and $4 \mathrm{mg} / \mathrm{mL}$, which were forced in combination with doxorubicin (in a co-spraying system) or alone.The experimental procedure was carried out with all the larvae descended from the crossing of females wts/TM3 with $m w h / m w h$ males. The results showed an anticarcinogenic activity of Gerioox®, since there was a statistically significant reduction $(p<0.05)$ in the tumor frequencies observed in the two highest tested concentrations of Gerioox® in association with $D X R$, when compared to the frequency of tumors in the positive control. It is concluded, therefore, that in the present experimental conditions, Gerioox® reduced the frequency of tumors induced by doxorubicin in $D$. melanogaster.

KEYWORDS: Anticarcinogenic, Drosophila melanogaster; Gerioox®.

\section{INTRODUÇÃO}

Toda informação genética está inscrita nos genes das células, em uma "memória química" conhecida como DNA. É por meio deste que os cromossomos transmitem as informações para o funcionamento da célula e, consequentemente, do organismo. A mesma, quando está normal, pode sofrer alterações no DNA, alterações estas conhecidas como mutações genéticas. Células cujo material genético foi alterado passam a receber instruções erradas para suas atividades, resultando em uma proliferação desorganizada, podendo culminar no aparecimento do câncer (JORDE et al., 2010).

O câncer é uma doença em que as células com alterações genéticas crescem de forma anormal, invadindo outros tecidos que perdem a função original (FENECH, 2002; FERRARI; TORRES, 2002). Diversas situações podem interferir na normalidade das células, tais como: agentes ambientais (tabagismo, raios ultravioletas, metais pesados), fatores químicos e biológicos, radicais livres, ou mesmo predisposição genética (FERNANDES; MELLO, 2008).

Os principais alvos da lesão genética referem-se a quatro classes de genes: os proto-oncogenes (genes promotores de crescimento), genes supressores do tumor (inibidores do crescimento), genes que regulam a morte celular programada (apoptose) e genes do reparo do DNA (DORNELAS, MORAES-FILHO, 2016). Uma quantidade substancial de evidências tem indicado os radicais livres como grandes responsáveis por alterações nesses genes, efeito que estimula a proliferação celular inadequada e, como consequência, o câncer (CRUZ, 2001).

Os efeitos dos antioxidantes em relação a numerosos tipos desta enfermidade já vêm sendo investigados (NETZEL et al., 2007). A terapia nutricional com antioxidantes concomitante à administração de drogas antineoplásicas apresenta vários benefícios ao tratamento oncológico. Além disso, a oferta de drogas antiblásticas (que combatem o câncer) associada às vitaminas antioxidantes como $\mathrm{A}, \mathrm{E}$ e $\mathrm{C}$, resulta em menores efeitos colaterais e permite que a continuidade do tratamento empregado não seja prejudicada, já que a toxicidade causada pelas drogas antineoplásicas é fator limitante desta terapia (CRUZ, 2001).

Esses agentes ainda protegem as células contra os efeitos dos radicais livres, pois inibem a oxidação de substratos provocada por estes, atuando em diferentes níveis de proteção (SOUSA et al., 2007). Desta forma, a terapêutica nutricional baseada na utilização de antioxidantes pode ampliar os conceitos da terapia oncológica atual e permitir melhores resultados quanto ao controle do câncer (KAPIL et al., 2004).

Os processos neoplásicos enquadram-se nas causas mais frequentes de morte em cães no Brasil, sendo que esta incidência aumenta na população geriátrica 
(FIGHERA et al., 2008). As neoplasias trazem uma série de consequências para a vida do animal, refletindo diretamente sobre a qualidade de vida do mesmo (OLIVEIRA et al., 2013).

Nesse contexto, a busca por medicamentos anticancerígenos tem aumentado com o propósito de encontrar tratamentos com maior efetividade e mais seletivos, ou que visem à descoberta de novas estratégias que impeçam a progressão da doença, minimizando os efeitos indesejados. Baseadas em avanços significativos na biologia do câncer, pesquisas buscam moléculas que atuem com mecanismos específicos e eficazes para cada tipo da enfermidade (ALTMANN; GERTSCH, 2007).

Algumas pesquisas relacionadas ao consumo do Gerioox® já foram realizadas com o intuito de verificar a eficácia na inibição do crescimento de alguns tipos de tumores, principalmente tumores gastrointestinais e reprodutores, entretanto, não existem, até o momento, comprovações sobre esse efeito (SUÁREZMAHECHA et al., 2012), nem avaliações sobre a administração simultânea de Gerioox® com agentes cancerígenos, como a doxorrubicina, utilizando a Drosophila melanogaster como organismo teste.

O Gerioox® é um medicamento composto por ômega 3, D-glucosamina, sulfato de condroitina, vitamina $E$, cobre, zinco e selenito de sódio. É indicado inicialmente como medicação geriátrica com efeitos antioxidantes, condroprotetores e dermatológicos para cães e gatos, de modo a melhorar a vitalidade e o estado geral de animais em idade avançada (CAVALLET; WOUK, 2007).

A Drosophila melanogaster, também conhecida como mosca da fruta, é empregada geneticamente desde 1909. Seu uso é recorrente em estudos, logo que apresenta características peculiares, tais como, ciclo de vida relativamente curto (cerca de 10 dias a $25^{\circ} \mathrm{C}$ ), fácil manutenção em laboratório, além de fornecer grande progênie (SNUSTAD; SIMMONS, 2006). Essa mosca possui considerável homologia genética com os mamíferos e adicionalmente um número significante de genes estudados (gene wts) na mosca confirmou ser homólogo de genes supressores de tumores (LATS1) em humanos e outros animais (GRIFFITHS et al., 2006).

Diante disso, este trabalho foi desenvolvido com o objetivo principal de avaliar o possível efeito anticarcinogênico do Gerioox®, por meio do teste para detecção de clones de tumores epiteliais (warts) em Drosophila melanogaster.

\section{MATERIAL E MÉTODOS}

\section{Agentes Químicos}

O Gerioox® é produzido pela Labyes Especialidades Veterinárias, Valinhos, São Paulo, Brasil. Cada comprimido possui: Ácidos Graxos Ômega 3: 0,2mL/ Glucosamina: 0,14g/ Gluconato de cobre: 0,003g / Gluconato de zinco: 0,02g / Selenito de sódio: $0,005 \mathrm{mg} /$ Sulfato de condroitina A: 0,15g/ Vitamina E: 0,05g e excipientes q.s.p. $1,8 \mathrm{~g}$ (GERIOOX®, 2009). Foi utilizado no presente experimento nas concentrações: 1 , 2 e $4 \mathrm{mg} / \mathrm{mL}$ (lote 004/15), baseadas em estudo desenvolvido por Capellari (2008), sobre a ação de diferentes componentes da matriz extracelular, tais como o sulfato de condroitina (componente do Gerioox®), sobre a ecto-5'nucleotidase, proliferação, adesão e migração celular na linhagem de células de glioma humano U138-MG.

$O$ agente indutor de tumor (controle positivo) foi o cloridrato de Doxorrubicina (DXR), denominado comercialmente como Adriblastina ${ }^{\circledR}$, pois 
apresenta atividade genotóxica e carcinogênica comprovada em Drosophila (ORSOLIN et al., 2012; VASCONCELOS et al., 2017). O mesmo foi preparado a partir da adição de $0,03538 \mathrm{~g}$ de Adriblastina ${ }^{\circledR}$ em $25 \mathrm{~mL}$ de água osmose reversa ultrapura, obtendo uma concentração de 0,4 mM (lote 5PL5023, CAS 25316-40-9). O laboratório Pfizer é o responsável pela produção deste medicamento, que é distribuído na forma de ampola com conteúdo de $50 \mathrm{mg}$. O acondicionamento é feito no Labcim (Laboratório de Citogenética e Mutagênese do Centro Universitário de Patos de Minas) sob condições ideais de temperatura e ausência de luz.

\section{Teste para detecção de clones de tumores epiteliais em Drosophila melanogaster}

A conservação evolutiva de genes supressores tumorais entre Drosophila e mamíferos tem levado a estudos na indução e no desenvolvimento de tumores nessas moscas. Diversos proto-oncogenes e supressores tumorais de mamíferos encontram-se também nesta espécie, o que pode ser um auxílio na compreensão do surgimento de cânceres em humanos e animais (EEKEN et al., 2002). A similaridade entre o gene supressor de tumor warts (wts) em Drosophila com o LATS1 em humanos já foram descritas. Quando deficiente este gene mostrou desenvolvimento de sarcomas em tecidos moles e tumores ovarianos (NISHIYAMA et al.,1999).

\section{Linhagens estoque}

O teste wts foi executado mediante o uso de duas linhagens mutantes de D. melanogaster (wts e $m w h$ ). A linhagem wts foi disponibilizada pelo Bloomington Drosophila Stock Center, da Universidade de Indiana, Estados Unidos, com o número de registro: Bloomington/7052, enquanto a linhagem $\mathrm{mwh} / \mathrm{mwh}$ foi fornecida pelo Dr. Ulrich Graf (Physiology and Animal Husbandry, Institute of Animal Science, ETH Zurich, Schwerzenbach, Switzerland).

Os estoques das mesmas são mantidos e cultivados no Laboratório de Citogenética e Mutagênese do Centro Universitário de Patos de Minas, armazenados em recipientes com meio de cultura próprio para $D$. melanogaster e conservados em uma incubadora, a temperatura de $25^{\circ} \mathrm{C}$ e aproximadamente $60 \%$ de umidade.

\section{Cruzamento}

Para obtenção de larvas heterozigotas (wts+/+mwh) foi realizado 0 cruzamento entre fêmeas virgens wts/TM3, Sb com machos $\mathrm{mwh} / \mathrm{mwh}$, em meio de cultura de banana. Após 48 horas, machos e as fêmeas foram colocados durante oito horas, em frascos contendo meio próprio para postura (fermento e açúcar), onde as fêmeas depositaram os ovos. As larvas de 72 horas, descendentes deste cruzamento foram todas tratadas com Gerioox® (nas três concentrações informadas anteriormente) e os respectivos controles: positivo (doxorrubicina) e negativo (água de osmose reversa).

\section{Procedimento experimental Tratamento}

Larvas de 72 horas resultantes do cruzamento acima descrito foram transferidas para frascos contendo $1,5 \mathrm{~g}$ de purê de batata (meio alternativo para Drosophila) e $5 \mathrm{~mL}$ de Gerioox® em três diferentes concentrações (1, 2 e $4 \mathrm{mg} / \mathrm{mL})$, além dos controles (separadamente). A associação entre Gerioox® e DXR foi efetuada em sistema de cotratamento (envolvendo exposição simultânea aos dois agentes) para análise da possível atividade anticarcinogênica do composto testado. 


\section{Análises das moscas}

Uma semana sequente ao tratamento, as moscas foram coletadas e armazenadas em frascos com etanol $70 \%$. Feito isso, estas foram separadas quanto ao fenótipo (apenas moscas portadoras de pelos finos e longos apresentam o gene wts, por isso, moscas com fenótipo de pelo curto e grosso são descartadas). Para a análise das moscas foram utilizadas lupas estereoscópicas e pinças entomológicas. Para o registro da frequência tumoral foi empregada uma planilha padrão, que separa quantitativamente a incidência de tumores nas regiões da asa, cabeça, corpo, olho, perna, halteres e o total por mosca, em cada concentração testada.

\section{Análise estatística}

As diferenças estatísticas entre as frequências de tumores das concentrações testadas e os controles foram calculadas utilizando o teste $U$, não paramétrico, de Mann-Whitney, empregando o nível de significância de 5\%.

\section{RESULTADOS E DISCUSSÃO}

Conforme observado na Tabela 1 foi possível verificar a frequência de tumores nos diferentes segmentos do corpo da $D$. melanogaster em indivíduos tratados com os controles (positivo e negativo) e as diferentes concentrações de Gerioox®. As larvas que não foram submetidas ao cotratamento com Doxorrubicina, mas somente expostas à solução de Gerioox®, nas concentrações de 1,2 e $4 \mathrm{mg} / \mathrm{mL}$ apresentaram frequências de 0,$23 ; 0,08$ e 0,07 tumores por mosca, respectivamente. Este resultado demonstra que o Gerioox®, quando comparado ao controle negativo, não provocou aumento na frequência tumoral, o que revela ausência de efeito carcinogênico do Gerioox® nas três concentrações testadas.

TABELA 1. Frequência de clones de tumores observados em Drosophila melanogaster, heterozigota para o gene supressor de tumor wts, tratadas com Gerioox® isolado ou em associação com doxorrubicina.

\begin{tabular}{|c|c|c|c|c|c|c|c|c|c|c|}
\hline \multicolumn{2}{|c|}{ Tratamentos } & \multirow[b]{2}{*}{$\begin{array}{c}\text { N. de } \\
\text { moscas }\end{array}$} & \multicolumn{7}{|c|}{ Número de tumores analisados } & \multirow[b]{2}{*}{$\begin{array}{c}\text { Frequência ( } \mathrm{N}^{\circ} \text { de } \\
\text { tumores/ mosca) }\end{array}$} \\
\hline $\begin{array}{c}\text { Gerioox® } \\
(\mathrm{mg} / \mathrm{mL})\end{array}$ & $\begin{array}{l}\text { DXR } \\
(\mathrm{mM})\end{array}$ & & Olho & Cabeça & Asa & Corpo & Perna & Halter & Total & \\
\hline 0 & 0 & 200 & 0 & 2 & 5 & 21 & 0 & 0 & 28 & 0,14 \\
\hline 1 & 0 & 200 & 0 & 6 & 5 & 33 & 1 & 1 & 46 & $0,23 \mathrm{~ns}^{*}$ \\
\hline 2 & 0 & 200 & 1 & 1 & 3 & 11 & 1 & 0 & 17 & $0,08^{*}$ \\
\hline 4 & 0 & 200 & 1 & 0 & 4 & 9 & 0 & 0 & 14 & $0,07^{*}$ \\
\hline 0 & 0,4 & 200 & 18 & 20 & 44 & 200 & 9 & 18 & 309 & $1,54^{*}$ \\
\hline 1 & 0,4 & 200 & 3 & 17 & 82 & 189 & 3 & 1 & 295 & $1,47 \mathrm{~ns}^{\star \star}$ \\
\hline 2 & 0,4 & 200 & 6 & 15 & 85 & 121 & 8 & 2 & 237 & $1,18^{\star \star}$ \\
\hline 4 & 0,4 & 200 & 0 & 0 & 8 & 40 & 0 & 0 & 48 & $0,24^{* *}$ \\
\hline
\end{tabular}

Diagnóstico estatístico de acordo com o Teste de Mann-Whitney. Nível de significância $p \leq$ 0,05 .

* Valor considerado diferente do controle negativo $(p \leq 0,05)$.

** Valor considerado diferente do controle positivo (DXR 0,4 mM) $(p \leq 0,05)$.

$\mathrm{ns}^{*}$, valores considerados não significativos, quando comparados com o controle negativo.

$\mathrm{ns}^{* *}$, valores considerados não significativos, quando comparados com o controle positivo.

DXR, doxorrubicina. 
Para o controle negativo utilizou-se água osmose reversa ultrapura, observando a frequência de 0,14 tumores por mosca. Esta discreta indução de tumores ocorre devido à predisposição genética do organismo teste. Já o controle positivo, 0,4 $\mathrm{mM}$ de $\mathrm{DXR}$, induziu uma frequência de 1,54 tumores por mosca (frequência significativamente superior à obtida no controle negativo). Tal fato evidencia que a linhagem responde à indução tumoral. A quimioterapia antineoplásica altera o DNA da célula tumoral com a finalidade de conter 0 crescimento. No entanto, como a substância age sistemicamente, as células saudáveis também podem sofrer tais alterações, ocorrendo efeito pró-tumoral (ALVES; NEPOMUCENO, 2012).

Pode-se perceber ainda, por meio dos resultados encontrados, que nas três concentrações estudadas de Gerioox®, foi constatado efeito anticarcinogênico, uma vez que ao analisar as concentrações associadas, em sistema de cotratamento com a DXR, observou-se diminuição de tumores quando comparado ao controle positivo isolado, embora o resultado tenha sido significativo apenas nas duas maiores concentrações $(p<0,05)$. Nessas concentrações as frequências totais de tumores foram, respectivamente, de 1,$47 ; 1,18$ e 0,24 tumores por mosca. É importante salientar que a frequência de tumores diminuiu com o aumento da dose utilizada, indicando que a resposta de inibição foi dose dependente.

Estudos anteriores corroboram com os resultados apresentados nessa pesquisa. Segundo Gus et al. (2006), o zinco, o cobre, a vitamina E e o selenito de sódio, encontrados no Gerioox®, são antioxidantes por excelência, e atuam eliminando os radicais livres, produtos naturais do metabolismo celular que quando não eliminados lesionam as células, provocando estresse oxidativo. Deste modo, a ação antioxidante pode ser justificada como um dos mecanismos pelos quais o Gerioox® reduziu a frequência de tumores, visto que um dos mecanismos de ação da Doxorrubicina é a geração de radicais livres.

Os danos oxidativos provocados nas células e tecidos têm sido relacionados com a etiologia de várias doenças, incluindo doenças degenerativas como cardiopatias, aterosclerose e problemas pulmonares, além disso, os danos causados pelos radicais livres no DNA desempenham papel importante nos processos de mutagênese e carcinogênese (BIANCHI; ANTUNES, 1999). Com o passar do tempo, a concentração de radicais livres na célula aumenta, e as defesas naturais antioxidantes tornam-se insuficientes e, com isso o uso de compostos antioxidantes identificados na dieta é um importante mecanismo de defesa contra os mesmos (SCOTTI; VELASCO, 2003; KENNEDY, 2006).

Zicker et al. (2008), após um estudo longitudinal com cães idosos, concluíram que os componentes do Gerioox® possuem expressiva atividade antioxidante, capaz de inibir a oxidação intracelular e das membranas celulares, diminuindo a morte celular provocada pelos radicais livres e, consequentemente, as doenças neoplásicas.

Os benefícios nutricionais e medicinais do ácido eicosapentaenoico (EPA) e docosahexaenoico (DHA), também conhecidos como ácidos graxos altamente insaturados ômega 3, presentes no Gerioox®, têm sido muito discutidos em artigos e conferências. Entre os efeitos fisiológicos estão a prevenção e tratamento de doenças cardiovasculares, hipertensão, inflamações em geral, asma, artrite, psoríase e vários tipos de câncer (SUÁREZ-MAHECHA et al., 2012).

Pode-se perceber que os antioxidantes presentes no Gerioox® podem trazer benefícios para a prevenção e tratamento de várias doenças, inclusive o câncer, pois possuem considerável atividade sequestradora de radicais hidroxila; 
além de agir como um quelante de ferro, evitando os possíveis distúrbios provocados por estes (KENNEDY, 2006).

Além do efeito antioxidante reconhecido do Gerioox®, estudos sugerem que o ômega 3 pode causar a restauração da via apoptótica que geralmente está prejudicada nos tecidos neoplásicos, ativando fosfatases proteicas envolvidas na inativação do Bcl-2 e na ativação da caspase-3, fatores que de maneira independente promovem o aumento da apoptose (NARAYANAN et al., 2001). Dessa forma, pode-se supor também, que o Gerioox®, ao induzir a apoptose em células tumorais, pode ter contribuído para a regressão na frequência de tumores observada no presente estudo.

A apoptose é um mecanismo biológico necessário para remover e retirar células mutantes ou moderadamente prejudicadas. É uma resposta de morte celular frente a agentes tóxicos e a desregulação é crucial no mecanismo de várias patologias, inclusive o câncer. Existem evidências de que o zinco e vitamina $E$, também presentes no Gerioox®, impedem que as células tumorais continuem o ciclo celular, interrompendo-o na fase $\mathrm{G1}$ e conduzindo as mesmas a apoptose (LAMSONL; BRIGNALL, 1999; TRUONG-TRAN et al., 2000).

Sendo assim, o potencial de ação das células malignas é reduzido e o potencial anticarcinogênico verificado no Gerioox® justifica-se em decorrência da significativa composição, que apresenta atividade antioxidante e apoptótica em células tumorais, podendo reduzir o dano oxidativo e a frequência de tumores induzidos pela doxorrubicina.

\section{CONCLUSÃO}

O teste para a detecção de clones de tumores em $D$. melanogaster foi eficiente para identificar o potencial anticarcinogênico do Gerioox®, justificando a utilização do mesmo, uma vez que as análises das concentrações associadas de Gerioox® com Doxorrubicina evidenciam redução na frequência de tumores em relação ao tratamento isolado com DXR. A presença de ômega 3, vitaminas e minerais na composição do Gerioox®, os quais possuem atividade antioxidante e apoptótica amplamente reconhecida, podem explicar tal efeito.

Este estudo oferece oportunidades para que demais pesquisas sejam desenvolvidas (envolvendo outras metodologias e outros organismos modelo), pois, a exploração das propriedades e efeitos biológicos do Gerioox® pode contribuir positivamente para maior qualidade de vida e promover avanços benéficos na saúde e prognóstico de animais acometidos por tumores malignos.

\section{REFERÊNCIAS}

ALTMANN, K. H.; GERTSCH, J. Anticancer drugs from nature-natural products as a unique source of new microtubule-stabilizing agents. Natural Product Reports, v.24, p. 327, 2007. Disponível em:< https://www.ncbi.nlm.nih.gov/pubmed/17390000>. doi: 10.1039/b515619j

ALVES, E. M.; NEPOMUCENO, J. C. Avaliação do efeito anticarcinogênico do látex do avelós (Euphorbia tirucalli), por meio do teste para detecção de clones de tumor (warts) em Drosophila melanogaster. Perquirere, Patos de Minas, v.9, n.2, p.125140, dez., 2012. Disponível em: http://perquirere.unipam.edu.br/documents/ 23456/57344/avaliacao-do-efeito-anticarcinogenico.pdf 
BIANCHI, M. L; ANTUNES, L, M. G. Radicais livres e os principais antioxidantes da dieta. Revista de Nutrição, Campinas, maio/ago., 1999. Disponível em: < http://dx.doi.org/10.1590/S1415-52731999000200001>. doi:0.1590/S1415-52731999 000200001

CAPELLARI, A. R. Efeito de diferentes componentes da matriz extracelular sobre a ecto-5'- nucleotidase, proliferação, adesão e migração celular na linhagem de células de glioma humano U138-MG. Dissertação (Mestrado em Bioquímica), Universidade Federal do Rio Grande do Sul, Porto Alegre, 2008. Disponível em: <http://www.lume.ufrgs.br/handle/10183/13847>.

CAVALLET, I. C. R; WOUK, A. F. P. F. Ácidos graxos essenciais comparados ao Gerioox® no tratamento do olho seco por facectomia em cães. Dissertação (Mestrado em Ciências Veterinárias), Curso de Pós Graduação em Ciências Veterinárias, Universidade Federal do Paraná, Curitiba, 2007. Disponível em: < http://acervodigital.ufpr.br/bitstream/handle/1884/14585/lzabel;jsessionid=EC5EC9A 3D16649D30A69B41433987AAA?sequence $=1>$

CRUZ, W. M. S. A terapia nutricional com vitaminas antioxidantes e o tratamento quimioterápico oncológico. Revista Brasileira de Cancerologia, Rio de Janeiro, v.47, n.3, p.303-308, 2001. Disponível em: http://homeopatia.yolasite.com/resources/PLANTAS_MEDICINAIS/terapia\%20nutrici onal.pdf

DORNELAS, C. A; MORAES-FILHOS, M. O. Carcinogênese experimental de bexiga e o índice de carcinogênese. Fortaleza: Imprensa Universitária, p.182, 2016. Disponível em:< http://www.repositorio.ufc.br/handle/riufc/19467>

EEKEN, J. C. J.; CLINK, I.; VEEN, B. L. V.; FERRO, W. Induction of epithelial tumors in Drosophila melanogaster heterozygous for the tumor suppressor gene wts. Enviromental and Molecular Mutagenesis, v. 40, p. 277-282, 2002. Disponível em: < https://www.ncbi.nlm.nih.gov/pubmed/12489118>. doi: 10.1002/em.10119

FENECH, M. Chromossomal biomarkers of genomic instability relevant to cancer. Drug Discovery Today, v.7, p. 1128-1137, 2002. Disponível em: < http://www.sciencedirect.com/science/article/pii/S1359644602025023>. doi:10.1016/ S1359-6446(02)02502-3

FERRARI, C. K.; TORRES, E. A. F. S. Novos compostos dietéticos com propriedades anticarcinogênicas. Revista brasileira de cancerologia, v. 48, p.37582, 2002.Disponível em:< http://www1.inca.gov.br/rbc/n_48/v03/pdf/artigo6.pdf>

FERNANDES, I.; MELLO, A. Entendendo e combatendo o câncer. Revista tema: Centro de Ensino Superior e Desenvolvimento, v.7, n.10/11, p. 02-11, Paraíba, 2008. Disponível em: <http://revistatema.facisa.edu.br/index.php/ revistatema/article/ viewArticle/1>. 
FIGHERA, R A.; SOUZA, T. M.; SILVA, M. G.; BRUM, J. S.; GRAÇA, D. L.; KOMMERS, G. D.; IRIGOYEN, L. F.; BARROS, C. S. L. Causas de morte e razões para eutanásia de cães da Mesorregião do Centro Ocidental Rio-Grandense (19652004). Pesquisa Veterinária Brasileira, v.28, n.4, p.223-230, 2008. Disponível em:< http://dx.doi.org/10.1590/S0100-736X2008000400005> doi: 10.1590/S0100-736X200 8000400005

GERIOOX®. TALARICO, J. São Paulo: Labyes do Brasil, [2009]. Bula de remédio. Registro: 9.451/2009.

GRIFFITHS, A. J. F; WESSLER, S. R; LEWONTIN, R. C; GELBART, W. M; SUZUKI, D. T; MILLER, J. H. Introdução à genética. 8.ed. Rio de Janeiro: Guanabara Koogan, 2006.

GUS, P. I.; BELLÓ-KLEIN, A.; LLESUY, S.; QUINTO, G. G.; MATOS, G. H.; BECHARA, S. J. Potencial Antioxidativo Da Lágrima De Adultos Jovens. Arquivos Brasileiros de Oftalmologia, v. 69, n. 4, p. 565-70, 2006. Disponível em:< http://dx.doi.org/10.1590/S0004-27492006000400020> doi: 10.1590/S000427492006000400020

JORDE, L. B; CAREY, J. C; BAMSHAD, M. J. Genética Médica. 4. ed. Rio de Janeiro: Elsevier, 2010. 350 p.

KAPIL, U.; SINGH, P.; SHUKLA, N. K.; PATHAK, P.; SINGH, R. Association of vitamin $A$, vitamin $C$ and zinc with laryngeal cancer. Indian Journal of Cancer, v.40, p.67-70, 2004. Disponível em: < http://www.indianjcancer.com/article.asp?issn=0019509X;year=2003; volume=40;issue =2; ;page=67;epage =70;aulast=Kapil $>$

KENNEDY, E. T. Evidence for nutritional benefits in prolonging wellness. The American Journal of Clinical Nutrition, v.83, n.2, 2006. Disponível em: <http://ajcn.nutrition.org/content/83/2/410S.full>

LAMSONL, D. W.; BRIGNALL, M. S. Antioxidants in cancer therapy; their actions and interactions with oncologic therapies. Alternative Medicine Review, v.4, p. 29 -304, 1999. Disponível em: <http://altmedrev.com/publications/5/2/152.pdf>

NARAYANAN, B. A.; NARAYANAN, N. K.; REDDY, B. S. Docosahexaenoic acid regulated genes and transcription factors inducing apoptosis in human colon cancer cells. International Journal of Oncology, v.19, p.1255 - 1262, 2001. Disponível em: < https://www.spandidos-publications.com/10.3892/ijo.19.6.1255?text=abstract> doi: 10.3892/ijo.19.6.1255

NETZEL, M.; NETZEL, G.; KAMMERER, D. R.; SCHIEBER, A.; CARLE, R.; SIMONS, L.; BITSCH, I.; BITSCH, R.; KONCZAK, I. Cancer cell antiproliferation activity and metabolism of black carrot anthocyanins. Innovative Food Science and Emerging Technologies, v.8, p.365-372, 2007. Disponível em: <http://www.sciencedirect.com/science/article/pii/S1466856407000392> doi: 10.1016/j.ifset.2007.03.011 
NISHIYAMA, Y.; HIROTA, T.; MORISAKI, T.; HARA, T.; MARUMOTO, T.; IADA, S.; MAKINO, K.; YAMAMOTO, H.; HIRAOKA, T.; KITAMURA, N.; SAYA, H. A human homolog of Drosophila warts supressor, $h$-warts, localized to mitotic apparatus and specifically phosphorylated during mitosos. Febs Letters, v.459, p.159-165, 1999. Disponível em: <http://www.sciencedirect.com/science/article/pii/S00145793990 12247> doi: 10.1016/S0014-5793(99)01224-7.

OLIVEIRA, K. M.; HORTA, R. S.; SILVA, C. M. O.; LAVOR, M. S. L. Principais síndromes paraneoplásicas em cães e gatos. Enciclopédia Biosfera, Goiânia, v.9, n.17, p. 2073-2088, 2013. Disponível em: <http://www.conhecer.org.br/enciclop/ 2013b/CIENCIAS\%20AGRARIAS/Principais.pdf>

ORSOLIN, P. C.; SILVA-OLIVEIRA, R. G.; NEPOMUCENO, J. C. Assessment of the mutagenic, recombinagenic and carcinogenic potential of orlistat in somatic cells of Drosophila melanogaster. Food and Chemical Toxicology, v.50, p.2598-2604, 2012. Disponível em: https://ac.els-cdn.com/S0278691512003390/1-s2.0S0278691512003390-main.pdf?_tid=98319c14-a2e5-11e7-a4ae-00000aacb35f\& acdnat=1506449444_ca93be96a43d2c1933f54359578030cb>doi:10.1016/j.fct.2012. 05.008

SCOTTI, L.; VELASCO, M. V. R. Envelhecimento cutâneo à luz da cosmetologia. São Paulo: Tecnopress, 2003.

SNUSTAD, D. P.; SIMMONS, M. J. Cellular reproduction and model genetic organisms. In: Principles of genetics. 4. ed. [S.I.]: Wiley, 2006. cap. 2, p. 17-41.

SOUSA, C. M.; SILVA, H. R. E.; VIEIRA-JR, G. M.; AYRES, M. C. C.; COSTA, C. L. S.; ARAÚJO, D. S.; CAVALCANTE, L. C. D.; BARROS, E. D. S.; ARAÚJO, P. B. M.; BRANDÃO, M. S.; CHAVES, M. H. Fenóis totais e atividade antioxidante de cinco plantas medicinais. Química nova, v.30, n.2, p. 351-355, 2007. Disponível em: <http://quimicanova.sbq.org.br/imagebank/pdf/Vol30No2_351_20-AR06044.pdf>

SUÁREZ-MAHECHA, H.; FRANCISCO, A. de; BEIRÃO, L. H.; BLOCK, J. M; SACCOL, A; PARDO-CARRASCO, S. A importância dos ácidos graxos poliinsaturados presentes em peixes. Boletim no Instituto de Pesca, v. 28, n.1, p.101- 110, 2012. Disponível em: <http://www.pesca.sp.gov.br/Suarez_mahecha. pdf>.

TRUONG-TRAN, A. Q.; HO, L.H.; CHAI F.; ZALEWSKI, P. D. Cellular zinc fluxes and the regulation of apoptpsis/gene-directed cell death. Journal of Nutrition, v.130, p.66-1459, 2000. Disponível em: <http://jn.nutrition.org/content/130/5/1459S.full>.

VASCONCELOS, M. A.; ORSOLIN, P. C.; SILVA-OLIVEIRA, R. G; NEPOMUCENO, J. C.; SPANÓ, M. A. Assessment of the carcinogenic potential of high intensesweeteners through the test for detection of epithelial tumor clones (warts) in Drosophila melanogaster. Food and Chemical Toxicology, v.101, p.1-7, 2017. Disponível em: < https://doi.org/10.1016/j.fct.2016.12.028> doi: 10.1016/j.fct. 2016.12.028 
ZICKER, S; ROFINA, J.; HEAD, E. Oxidative stress, aging and CNS disease in the canine model of human brain aging. Veterinary Clinics of North America: Small Animal Practice, v. 38, p.167-178, 2008. Disponível em: < https://doi.org/10.1016/j. cvsm.2007.10.002> doi: 10.1016/j.cvsm.2007.10.002 